\title{
Sex-Biased Dispersal and the Speed of Two-Sex Invasions
}

\author{
Tom E. X. Miller, ${ }^{1, \star}$ Allison K. Shaw, ${ }^{2, \star}$ Brian D. Inouye, ${ }^{3}$ and Michael G. Neubert ${ }^{4, \dagger}$ \\ 1. Department of Ecology and Evolutionary Biology, MS-170, Rice University, Houston, Texas 77005; 2. Department of Ecology and \\ Evolutionary Biology, Princeton University, Princeton, New Jersey 08544; 3. Department of Biological Science, Florida State University, \\ Tallahassee, Florida 32306; 4. Biology Department, MS 34, Woods Hole Oceanographic Institution, Woods Hole, Massachusetts 02543 \\ Submitted September 13, 2010; Accepted February 4, 2011; Electronically published April 7, 2011 \\ Online enhancement: appendix.
}

\begin{abstract}
Population models that combine demography and dispersal are important tools for forecasting the spatial spread of biological invasions. Current models describe the dynamics of only one sex (typically females). Such models cannot account for the sexrelated biases in dispersal and mating behavior that are typical of many animal species. In this article, we construct a two-sex integrodifference equation model that overcomes these limitations. We derive an explicit formula for the invasion speed from the model and use it to show that sex-biased dispersal may significantly increase or decrease the invasion speed by skewing the operational sex ratio at the invasion's low-density leading edge. Which of these possible outcomes occurs depends sensitively on complex interactions among the direction of dispersal bias, the magnitude of bias, and the relative contributions of females and males to local population growth.
\end{abstract}

Keywords: integrodifference equations, invasion speed, mating functions, marriage squeeze, sex-biased dispersal, spatial spread.

\section{Introduction}

Population dynamics play out in time and space. Understanding and predicting population trajectories in both dimensions are core objectives in the study of biological invasions, a term we use broadly to describe population expansion into an unoccupied region. Conceptually, studies of biological invasions can shed light onto such problems as spatial pattern formation (Maron and Harrison 1997; Wilson et al. 2002), the formation of range boundaries (Keitt et al. 2001), and colonization following primary succession (Fagan et al. 2005). Practically, the ability to predict population spatial spread could aid in management efforts for threatened species recolonizing formerly occupied habitat (Tinker et al. 2008), species whose ranges are shifting in response to global climate change (Potapov and Lewis 2004; Crozier and Dwyer 2006; Zhou and Kot 2010), exotic species invading novel environments (Andow

\footnotetext{
* These authors contributed equally to the article.

$\dagger$ Corresponding author; e-mail: mneubert@whoi.edu.
}

Am. Nat. 2011. Vol. 177, pp. 549-561. (C) 2011 by The University of Chicago. 0003-0147/2011/17705-52467\$15.00. All rights reserved. DOI: $10.1086 / 659628$ et al. 1990; Neubert and Parker 2004; Buckley et al. 2005; Miller and Tenhumberg 2010), and biological control agents intended to slow or halt the spread of invasive pests (Sharov and Liebhold 1998; Fagan et al. 2002).

The theoretical framework for biological invasions consists of mathematical models that couple local demography with individual movement. The demographic component determines, through birth and survival, the number of propagules at a given location, and the dispersal component redistributes propagules throughout space. Together, local population growth and individual movement often generate a wave of organisms that advances through space. A large body of theory (Skellam 1951; Okubo 1980; Kot et al. 1996; Neubert and Caswell 2000; Hastings et al. 2005) indicates that (in the absence of Allee effects) the velocity of the traveling wave is governed by the asymptotic dynamics of the population at its low-density leading edge.

As in the rest of population ecology, most of the currently available models for biological invasions explicitly track the densities of only one sex. One-sex models are well suited to asexual or hermaphroditic species, including most plants. However, the application of one-sex invasion theory to dioecious species is complicated by the fact that dispersal may be sex biased, with males and females moving different distances.

To understand the potential significance of sex-biased dispersal in spatial spread, first imagine a closed local population (no spatial dynamics). Barring any demographic differences between females and males, the sex ratio of mating individuals, or the operational sex ratio, will reflect the sex ratio at birth. The birth sex ratio, in turn, is constrained to an evolutionarily stable value (typically 0.5 due to the frequency-dependent advantage of producing the rarer sex; Fisher 1930). Thus, in the absence of spatial dynamics, the operational sex ratio should remain roughly constant, and from a population dynamics perspective, no information is gained by tracking the densities of both females and males; one sex tells the whole story.

The introduction of spatial dynamics changes the story. When individuals of different sexes have different dispersal 
distributions, the operational sex ratio can become skewed at some locations. This skew would be most severe far in front of an invasion wave, where the sex with the shorter dispersal distance would be relatively scarce or absent. ${ }^{1}$ Since in most cases it is the population dynamics in exactly these locations that determine the invasion speed, one may reasonably surmise that sex-biased dispersal should affect invasion speed. Thus, the application of one-sex theory to two-sex invasions is likely to be accurate under at least one of two rarely acknowledged conditions: (1) females and males share an identical dispersal kernel (in which case, as in the strictly local scenario, no information is gained by tracking both sexes), or (2) the operational sex ratio has no effect on the low-density population growth rate.

In nature, these conditions may be rarely met. First, sex-biased dispersal is a common phenomenon in animals (see fig. 1 for three examples from vertebrates). Natal dispersal distances are generally male biased (i.e., males travel farther) in mammals (Greenwood 1980; Dobson 1982) and female biased in birds (Clarke et al. 1997; Dale 2001). Our review of the dispersal literature for insects and birds, taxonomic groups that include many important invaders, indicated that both male- and female-biased dispersal are common (fig. 2). Second, little is known empirically regarding effects of skew in the operational sex ratio on population growth rates. It is often assumed for convenience that females dominate population growth dynamics, with fertility independent of male availability (Rankin and Kokko 2007). However, the assumption of frequencyindependent dynamics is almost certainly incorrect in extreme cases (when the population is nearly $100 \%$ female or male), and models of two-sex population dynamics indicate that even a small departure from a $1: 1$ sex ratio can have a large effect on the birth rate (Caswell and Weeks 1986; Legendre et al. 1999). These observations suggest that existing one-sex invasion theory may be inadequate for understanding and predicting the spatial dynamics of many biological invasions.

In the following sections of this article we construct and analyze a spatially explicit two-sex population model to examine how sex-biased dispersal and sex-ratio-dependent population dynamics interact to influence the speed of biological invasions. Because the effects of the sex ratio on demography may depend on the social mating system, we consider biological invasions by both monogamous and nonmonogamous populations that differ in the degree to which recruitment is limited by females or males. Our model combines two established mathematical approaches: integrodifference models for population spatial spread (Kot et al. 1996; Kot 2002) and pair formation

\footnotetext{
${ }^{1}$ In the literature dealing with human demography, an extreme skew in the sex ratio is said to generate a "marriage squeeze" (Schoen 1983).
}
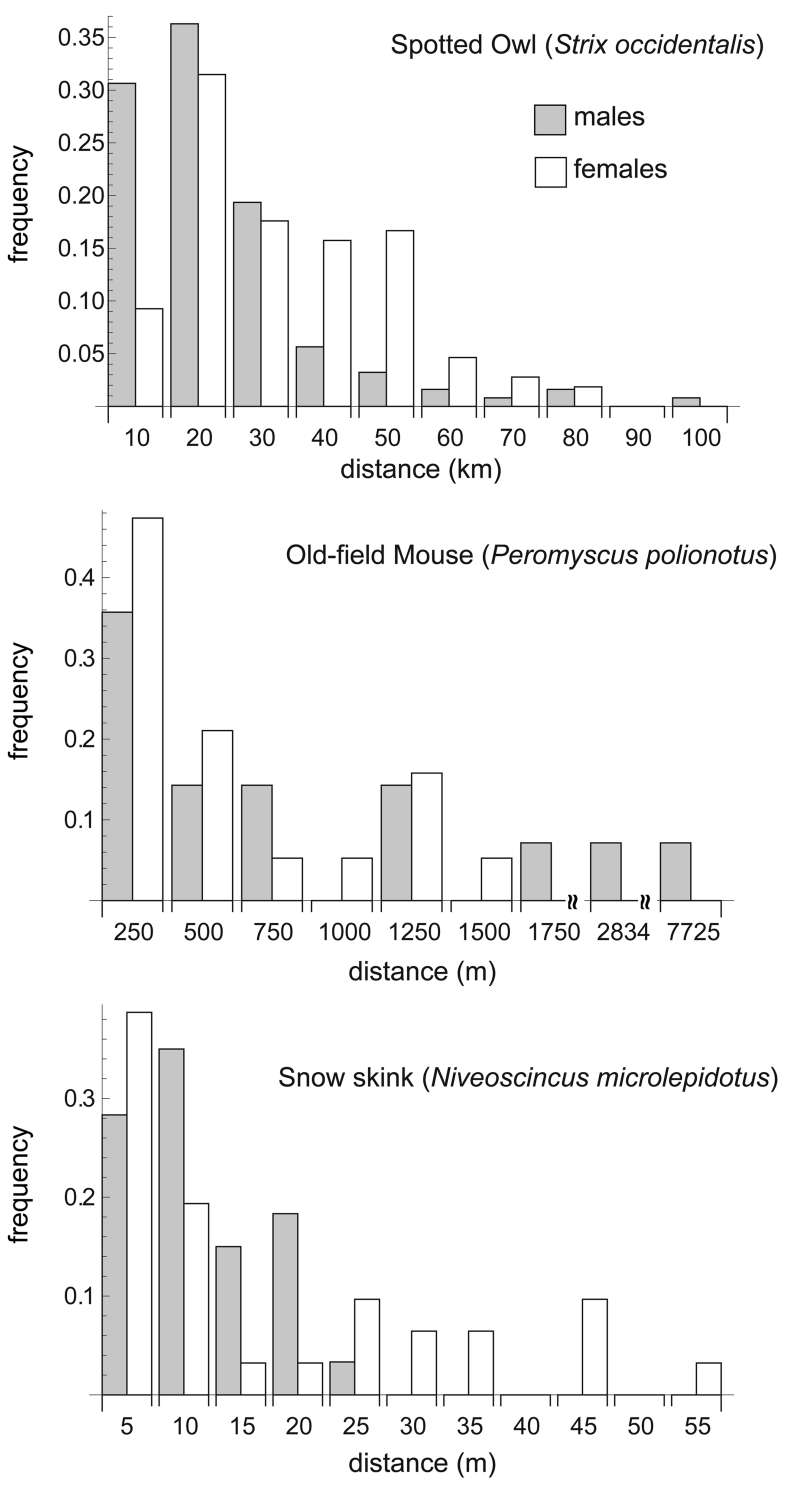

Figure 1: Sex-biased dispersal distributions of the spotted owl (124 males, 108 females; Franklin et al. 1996), old-field mice (14 males, 19 females; Smith 1968), and snow skinks (60 males, 31 females; Olsson and Shine 2003).

models for local birth rates under different mating systems (Caswell and Weeks 1986). We derive an upper bound on the invasion speed that this model predicts and, with evidence from numerical simulations, conjecture that this upper bound is the exact asymptotic invasion speed. The formula for the two-sex invasion speed is not based on a linearization of the model at low densities as it is for singlespecies one-sex models. Rather, it is based on a nonlinear approximation, for which we are able to construct traveling wave solutions. 

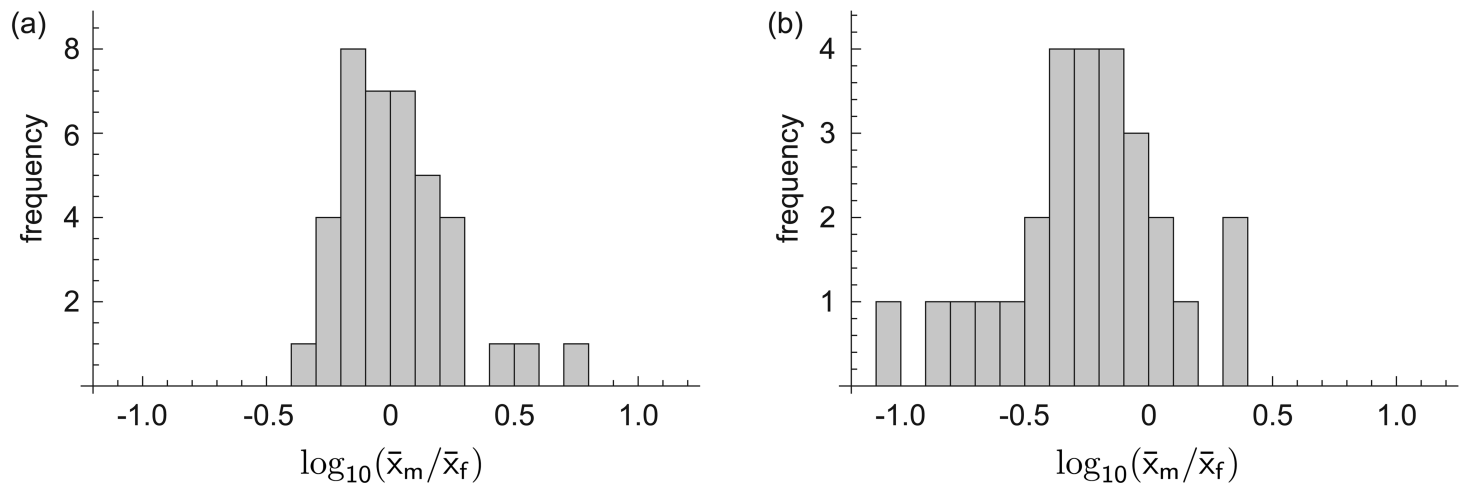

Figure 2: The logarithm of the ratio of the mean male dispersal distance $\left(\bar{x}_{\mathrm{m}}\right)$ to the mean female dispersal distance $\left(\bar{x}_{\mathrm{f}}\right)$ from studies of 38 insect species $(a)$ and 27 bird species $(b)$. See tables A1 and A2 in the online edition of the American Naturalist for data and references. For species with multiple dispersal estimates (e.g., from different sites or years), we used sex-specific species averages.

\section{Integrodifference Models}

\section{One Sex}

A useful foundation for the construction of a two-sex invasion model is the simpler one-sex integrodifference equation model (Kot and Schaffer 1986; Kot 1992):

$$
n_{t+1}(x)=\int_{-\infty}^{\infty} k(x, y) G\left(n_{t}(y)\right) d y .
$$

This model describes the change in population density $n_{t}(x)$ between times $t$ and $t+1$ that results from two processes operating sequentially. First, new individuals are produced at each location $y$ as given by the nonlinear function $G\left(n_{t}(y)\right)$. Next, these new individuals move. Their redistribution through space is prescribed by the dispersal kernel $k(x, y)$, a probability density function for individual displacements from location $y$ to location $x$. If the probability of moving from $y$ to $x$ does not depend on the exact values of $y$ and $x$ but only on the (signed) distance between them $(x-y)$, equation (1) takes the form of a convolution:

$$
n_{t+1}(x)=\int_{-\infty}^{\infty} k(x-y) G\left(n_{t}(y)\right) d y .
$$

Under model (2), a small population initially concentrated around a single location will grow and eventually spread as a wave as long as the low-density growth rate is positive (i.e., as long as $G^{\prime}(0)>1$ ). Under further conditions prescribed by Weinberger (1978, 1982; the most stringent of which is the absence of an Allee effect), the asymptotic speed of this wave is the same as the wave speed of the low-density linear approximation to model (2):

$$
n_{t+1}(x)=G^{\prime}(0) \int_{-\infty}^{\infty} k(x-y) n_{t}(y) d y .
$$

The speed is given by the formula

$$
\tilde{c}=\min _{s}\left[\frac{1}{s} \ln \left(G^{\prime}(0) M(s)\right)\right],
$$

where $M(s)$ is the moment-generating function of the dispersal kernel:

$$
M(s)=\int_{-\infty}^{\infty} k(x) e^{s x} d x
$$

\section{Two Sexes}

We now build on model (2) to account for two sexes, their mating behavior, and possible differences between the sexes in dispersal behavior. Let $m_{t}(x)$ and $f_{t}(x)$ be the population densities of males and females at time $t$. In our model, three processes operate sequentially to determine the distribution of the population at time $t+1$. First, males and females mate. The mating density - the number of matings per unit distance at location $x$-is given by the mating function $\mathfrak{B}\left(m_{t}(x), f_{t}(x)\right){ }^{2}$

Next, each mating produces offspring. At low population densities, each mating produces $\mu$ males and $\phi$ females

\footnotetext{
${ }^{2}$ In the literature on human demography, the function $\mathfrak{B}$ is called the "marriage function" (Iannelli et al. 2005).
} 
that survive to become adults in the next generation. At higher population densities, intraspecific competition reduces the numbers of surviving offspring by the fraction $g$. For simplicity, we assume $g$ is a function of the weighted population density $N_{t}=a_{\mathrm{m}} m_{t}+a_{\mathrm{f}} f_{t}$, with nonnegative constants $a_{\mathrm{m}}$ and $a_{\mathrm{f}}$ not both 0 . Finally, the surviving individuals disperse, males according to the dispersal kernel $k_{\mathrm{m}}(x)$ and females according to the dispersal kernel $k_{\mathrm{f}}(x)$. Concatenating the mating, reproduction and survival, and dispersal processes gives

$$
\begin{aligned}
m_{t+1}(x) & =\int_{-\infty}^{\infty} k_{\mathrm{m}}(x-y)\left[\mu g\left(N_{t}(y)\right) \mathfrak{B}\left(m_{t}(y), f_{t}(y)\right)\right] d y, \\
f_{t+1}(x) & =\int_{-\infty}^{\infty} k_{\mathrm{f}}(x-y)\left[\phi g\left(N_{t}(y)\right) \mathfrak{B}\left(m_{t}(y), f_{t}(y)\right)\right] d y .
\end{aligned}
$$

Model (6) can be generalized to accommodate various mating systems. Rosen (1983) imagined a polygynous mating system in which males mate with "harems" of females of size $h \geq 1$. The total number of harems is then $f / h$, and the density of matings between adult males and harems is $\mathfrak{B}(m, f / h)$. The numbers of male and female offspring produced by a harem are $\mu h$ and $\phi h$, respectively, and system (6) becomes

$$
\begin{aligned}
& m_{t+1}(x)=\int_{-\infty}^{\infty} k_{\mathrm{m}}(x-y)\left[\mu h g\left(N_{t}(y)\right) \mathfrak{B}\left(m_{t}(y), \frac{f_{t}(y)}{h}\right)\right] d y \\
& f_{t+1}(x)=\int_{-\infty}^{\infty} k_{\mathrm{f}}(x-y)\left[\phi h g\left(N_{t}(y)\right) \mathfrak{B}\left(m_{t}(y), \frac{f_{t}(y)}{h}\right)\right] d y
\end{aligned}
$$

Monogamous and polyandrous mating systems can be accommodated by setting $h=1$ or $h<1$, respectively.

The mating function at the heart of model (7) could take many forms. According to Caswell (2001), most mathematical demographers agree that

(1) $\mathfrak{B}(m, f)$ should be well defined and nonnegative whenever its arguments are nonnegative;

(2) $\mathfrak{B}(m, f)$ should be a homogeneous function of $m$ and $f$ of first degree (i.e., for any constant $a$, $\mathfrak{B}(a m, a f)=a \mathfrak{B}(m, f)$

(3) $\mathfrak{B}(m, f)$ should be a nondecreasing function of its arguments (i.e., $\partial \mathfrak{B} / \partial m \geq 0$ and $\partial \mathfrak{B} / \partial f \geq 0$ for all $m \geq 0$ and $f \geq 0$ ); ceteris paribus, more males or females results in more matings; and

(4) in the absence of either sex there are no matings (and hence no births): $\mathfrak{B}(m, 0)=\mathfrak{B}(0, f)=\mathfrak{B}(0,0)=0$ (see also Iannelli et al. 2005).

Numerous mating functions that satisfy axioms 1-4 appear in the demographic literature (Caswell 2001). Most of these are members of the family of weighted power means (also known as Hölder means; Bullen 2003) that was suggested by Hadeler (1989). This family has the form

$$
\mathfrak{B}(m, f)=\left[w m^{p}+(1-w) f^{p}\right]^{1 / p},
$$

with $0 \leq w \leq 1$ and $p<0$. For equal weights $(w=1 / 2)$, this family includes the geometric mean

$$
\mathfrak{B}_{\text {geo }}(m, f)=\sqrt{m f}
$$

in the limit as $p \rightarrow 0$, the harmonic mean

$$
\mathfrak{B}_{\text {har }}(m, f)=\frac{2 m f}{m+f}
$$

for $p=-1$, and the minimum

$$
\mathfrak{B}_{\min }(m, f)=\min (m, f)
$$

in the limit as $p \rightarrow-\infty$ (Hardy et al. 1952). The standard female-dominant model

$$
\mathfrak{B}_{\text {fem }}(m, f)=f
$$

(which does not satisfy axiom 4 ) is a weighted power mean with $w=0$.

To derive a formula for the invasion speed, the specific form of the mating function is not important as long as it satisfies the four axioms above. Later, in "Numerical Simulations," we will compare the invasion speeds generated by two-sex mating functions from the family (8) with the speeds predicted by the standard female-dominant function (12), which is equivalent to the one-sex model (2). Note that as the harem size increases, the density of mated females, $h \mathfrak{B}(m, f / h)$, approaches the femaledominant mating model. This is true for all members of the family (8) with $p<0$ except for the geometric mean (9).

\section{The Invasion Speed}

For a large class of dispersal kernels and initial conditions, model (7) generates an invasion if the numbers of male and female offspring produced per mating ( $\mu$ and $\phi)$ are large enough. That is, a small population, initially concentrated in a restricted area, grows, expands, and takes the shape of an advancing wave (fig. 3, left column). As time proceeds, the speed of the wave asymptotically 

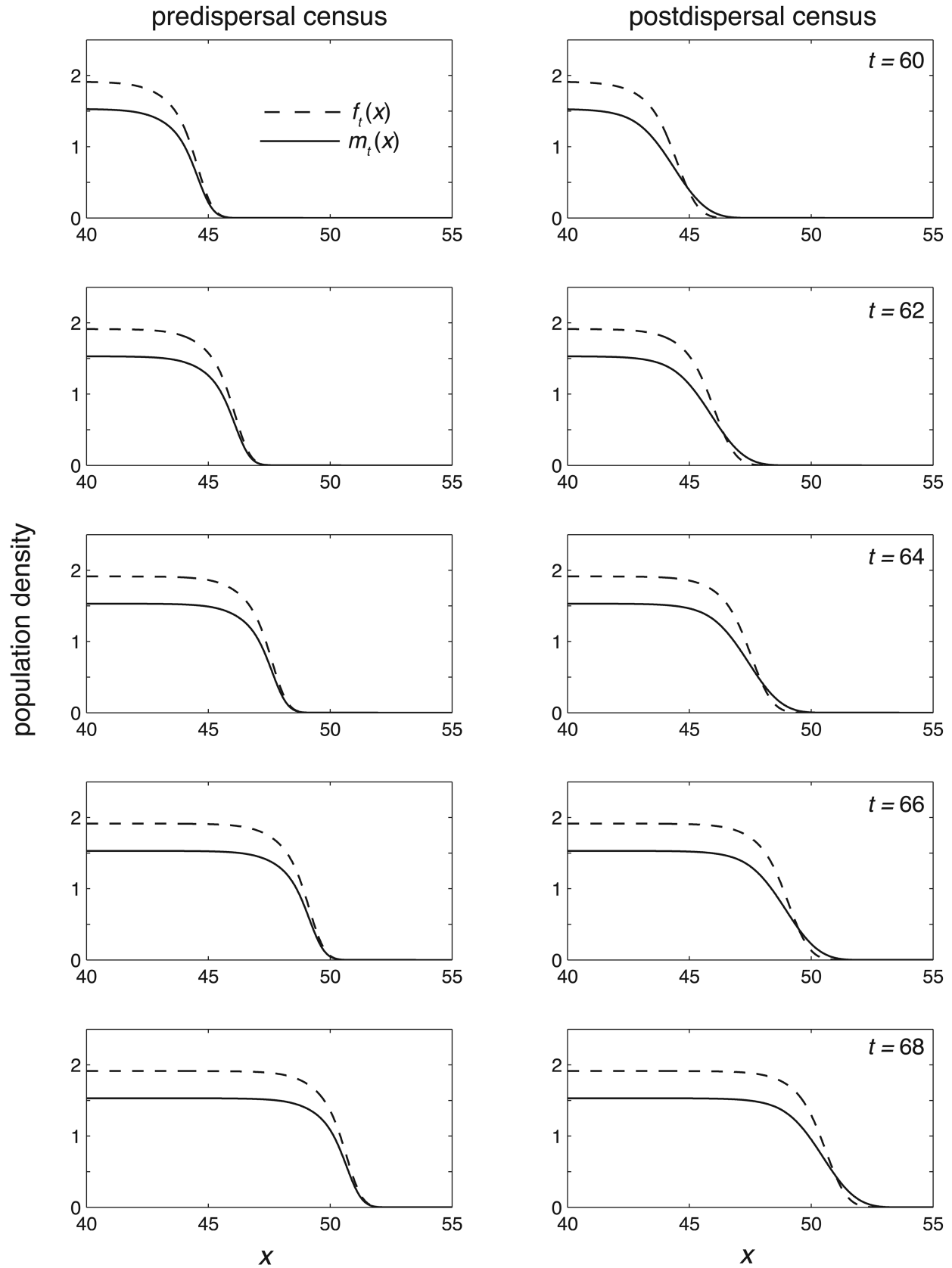

Figure 3: Invasion waves generated by the postdispersal-census model (eq. [18]) and the predispersal model (eq. [19]). For this example we used equation (14) as the density-dependent survival function, with $a_{\mathrm{m}}=a_{\mathrm{f}}=a=1, \mu=8$, and $\phi=10$. For the dispersal kernels we used the two-sided power distribution equation (27), with $n=2$ for each sex. The maximum dispersal distance was male biased $\left(b_{\mathrm{m}}=2, b_{\mathrm{f}}=1\right)$. We set the initial population densities for both sexes to 0.5 if $|x|<0.1$ and to 0 otherwise.

approaches a constant value. It is this asymptotic speed that we call the "invasion speed." In this section, we derive an upper bound on the invasion speed. We conjecture that this upper bound is the exact asymptotic speed for many biologically relevant initial conditions and dispersal kernels. Our conjecture is supported by the results of numerical simulations described in "Numerical Simulations."

Our derivation rests in part on further assumptions regarding the density-dependent function $g$ and the mating 
function $\mathfrak{B}$. First, we assume the absence of positive density dependence, so that

$$
\begin{gathered}
g(0)=1, \\
g^{\prime}(N) \leq 0,
\end{gathered}
$$

for all $N \geq 0$; that is, increases in population density decrease survival. An example of such a function (one that we will use in simulations that follow) is

$$
g(N)=\frac{a}{a+N} .
$$

With our assumption (13), one can show that the population will grow from low densities only if

$$
\mathfrak{B}(h \mu, \phi)>1 \text {. }
$$

An invasion will not proceed unless condition (15) is satisfied; we will assume condition (15) holds.

Finally, we will restrict our attention to purely compensatory population growth; that is, we will require that

$$
\begin{gathered}
\frac{\partial}{\partial m}[g(N) \mathfrak{B}(h m, f)]>0, \\
\frac{\partial}{\partial f}[g(N) \mathfrak{B}(h m, f)]>0,
\end{gathered}
$$

for all $m>0$ and $f>0$. We expect, however, that the results we present below will be true in the case of overcompensatory growth as well.

Using the fact that $\mathfrak{B}$ is homogeneous and defining the convolution operator " $*$ " as

$$
p(x) * q(x)=\int_{-\infty}^{\infty} p(x-y) q(y) d y,
$$

system (7) can be rewritten as

$$
\begin{aligned}
m_{t+1}(x) & =k_{\mathrm{m}}(x) *\left[\mu g\left(N_{t}(x)\right) \mathfrak{B}\left(h m_{t}(x), f_{t}(x)\right)\right], \\
f_{t+1}(x) & =k_{\mathrm{f}}(x) *\left[\phi g\left(N_{t}(x)\right) \mathfrak{B}\left(h m_{t}(x), f_{t}(x)\right)\right] .
\end{aligned}
$$

In this formulation, the population is counted just after dispersal. If, on the other hand, we were to census the population just before dispersal, we would have the alternative formulation

$$
\begin{aligned}
m_{t+1}(x)= & \mu g\left(a_{\mathrm{m}} m_{t}(x) * k_{\mathrm{m}}(x)+a_{\mathrm{f}} f_{t}(x) * k_{\mathrm{f}}(x)\right) \\
& \times \mathfrak{B}\left(h m_{t}(x) * k_{\mathrm{m}}(x), f_{t}(x) * k_{\mathrm{f}}(x)\right), \\
f_{t+1}(x)= & \phi g\left(a_{\mathrm{m}} m_{t}(x) * k_{\mathrm{m}}(x)+a_{\mathrm{f}} f_{t}(x) * k_{\mathrm{f}}(x)\right) \\
& \times \mathfrak{B}\left(h m_{t}(x) * k_{\mathrm{m}}(x), f_{t}(x) * k_{\mathrm{f}}(x)\right) .
\end{aligned}
$$

This later form is useful because it shows that, whatever the initial densities $m_{0}(x)$ and $f_{0}(x)$,

$$
m_{t}(x)=\frac{\mu}{\phi} f_{t}(x)
$$

for $t \geq 1$ when measured just before dispersal. System (19) can then be reduced to the single equation

$$
\begin{aligned}
f_{t+1}(x)= & g\left[\left(\frac{\mu}{\phi}\right) a_{\mathrm{m}} f_{t}(x) * k_{\mathrm{m}}(x)+a_{\mathrm{f}} f_{t}(x) * k_{\mathrm{f}}(x)\right] \\
& \times \mathfrak{B}\left(h \mu f_{t}(x) * k_{\mathrm{m}}(x), \phi f_{t}(x) * k_{\mathrm{f}}(x)\right) .
\end{aligned}
$$

Whether we choose the traditional postdispersal census model (18) or the predispersal census model (21), the qualitative dynamics are the same (fig. 3): an invasion wave is formed. The speed of the wave is the same under both models, but model (21) is easier to analyze.

Because of our assumptions about the function $g$, one can show that solutions to the recursion (21) are bounded above by solutions of

$$
f_{t+1}(x)=\mathfrak{B}\left(h \mu f_{t}(x) * k_{\mathrm{m}}(x), \phi f_{t}(x) * k_{\mathrm{f}}(x)\right)
$$

(see appendix for additional details). A population governed by equation (21) cannot spread faster than one governed by equation (22) with the same initial condition.

As does model (21), the nonlinear recursion (22) has traveling wave solutions. We find these by substituting $f_{t}(x-c)$ for $f_{t+1}(x)$. After dropping the $t$ we have

$$
f(x-c)=\mathfrak{B}\left(h \mu f(x) * k_{\mathrm{m}}(x), \phi f(x) * k_{\mathrm{f}}(x)\right) .
$$

This nonlinear equation has solutions of the form $f(x)=a e^{-s x}$, with $a>0$. Substitution of this form into equation (23) yields a dispersion relation, which gives the speed of the wave, $c$, as a function of its shape $s$ :

$$
c(s)=\frac{1}{s} \ln \mathfrak{B}\left(h \mu M_{\mathrm{m}}(s), \phi M_{\mathrm{f}}(s)\right),
$$

where $M_{\mathrm{m}}(s)$ and $M_{\mathrm{f}}(s)$ are the moment-generating functions of the male and female dispersal kernels (cf. equation [5]).

Equation (24) gives the speed of invasion for a population with an exponential distribution in space. Such an initial population unrealistically has individuals everywhere and an arbitrarily large population density for large negative $x$. Any real invasion, on the other hand, begins with a population that has finite range and finite size, and therefore, $f_{0}(x) \leq a e^{-s x}$ for sufficiently large $a$. Since $\mathfrak{B}$ is a nondecreasing function, one can show that if $f_{0}(x) \leq$ $a e^{-s x}$, then $f_{t}(x) \leq a e^{-s(x-c(s) t)}$ for $t>0$ (see appendix). Therefore, populations with realistic initial conditions cannot spread faster than even the slowest exponential-front traveling wave solution of recursion (22). And since solutions to model (21) are bounded above by solutions to recursion (22), the speed of invasion for model (21) (and therefore model [18]) must be less than or equal to 


$$
\begin{aligned}
\tilde{c} & =\min _{s} c(s) \\
& =\min _{s}\left[\frac{1}{s} \ln \mathfrak{B}\left(h \mu M_{\mathrm{m}}(s), \phi M_{\mathrm{f}}(s)\right)\right] .
\end{aligned}
$$

Numerical simulations (described next) suggest that for most realistic initial conditions and dispersal kernels, $\tilde{c}$ is in fact the exact asymptotic speed. However, there are combinations of dispersal kernels and initial conditions for which equation (25) will produce a positive speed but for which the invasion will not materialize. For the postdispersal model (18), if the initial distributions of the two sexes do not overlap, $\mathfrak{B}=0$ for all $x$ and the invasion immediately fails. If, on the other hand, the two sexes have identical initial distributions, say, $m_{0}(x)=f_{0}(x)=1$ for $|x|<1$ and 0 otherwise, the invasion may still not proceed if the dispersal kernels of males and females are such that males and females do not co-occur after dispersal. This would be the case if, for example,

$$
\begin{aligned}
& k_{\mathrm{m}}(x)=\left\{\begin{array}{ll}
\frac{1}{2} & \text { if }|x| \leq 1 \\
0 & \text { otherwise }
\end{array},\right. \\
& k_{\mathrm{f}}(x)=\left\{\begin{array}{ll}
\frac{1}{2} & \text { if } 4 \leq|x| \leq 5 \\
0 & \text { otherwise }
\end{array} .\right.
\end{aligned}
$$

Such arguments lead us to the conclusion that a necessary condition for an invasion to proceed is the existence of some time $t^{*}$ beyond which the distributions of males and females always overlap. Exactly how this condition mathematically constrains the initial conditions and dispersal kernels remains, for the time being, an open problem.

\section{Numerical Simulations}

We tested the invasion speed formula (25) by simulating model (21) using the density-dependent survival model (14) and the harmonic mean mating function (10), along with symmetric two-sided power distributions (Van Dorp and Kotz 2002; Kotz and Van Dorp 2004):

$$
k(x)= \begin{cases}\frac{n}{2 b}\left(1-\frac{|x|}{b}\right)^{n-1} & |x| \leq b, \\ 0 & |x|>b\end{cases}
$$

with scale parameter $b>0$ and shape parameter $n>0$ for the dispersal kernels.

The two-sided power distribution can take many shapes. For large $n$, the distribution is concentrated around $x=$ 0 . For small $n$, it is bimodal, with peaks at the maximum dispersal distance $b$. Special cases include the triangle distribution for $n=2$ and the uniform for $n=1$. We set $b_{\mathrm{m}}$ as the maximum dispersal distance for males and $b_{\mathrm{f}}$ as the maximum dispersal distance for females. The momentgenerating function for the two-sided power distribution is

$$
M(s)=\frac{n}{2}[\nu(b s, n)+\nu(-b s, n)]
$$

where $\nu(z, n)=e^{z} z^{-n}[\Gamma(n)-\Gamma(n, z)]$ and where $\Gamma(n)$ and $\Gamma(n, z)$ are the gamma function and the incomplete gamma function, respectively (Abramowitz and Stegun 1972).

As is clear from figure 4, the predicted speeds and simulation speeds match. We have tested equation (25) for other mating functions and other dispersal kernels that comply with the conditions described in "Integrodifference Models." In every case in which an invasion proceeded, the predicted and simulated speeds agreed. Emboldened by these results, we use equation (25) to examine how the invasion speed changes as a function of key model parameters.

The invasion speed is 0 for sufficiently small values of the harem size $h$ and the number of male offspring per mating $\mu$ (fig. 4). These thresholds are determined by inequality (15). The invasion speed increases monotonically with $h$ and $\mu$. As either of these parameters becomes large, the effective sex ratio before dispersal is skewed toward males, and females become the limiting sex. As a result, the invasion speed for the two-sex model approaches the invasion speed of the female-dominant model in the limit as $h$ or $\mu$ goes to infinity.

Invasion speed similarly increases with the maximum male dispersal distance $b_{\mathrm{m}}$. As males disperse farther, females again become limiting, and the invasion speed approaches the speed of the female-dominant model. In contrast to $h$ and $\mu$, however, the the invasion speed is positive for all positive $b_{\mathrm{m}}$.

\section{Invasion Speed and Dispersal Bias}

To explore the effects of differences in dispersal between males and females, we define the dispersal bias, $\psi$, as the logarithm of the ratio of the mean male dispersal distance to the mean female dispersal distance. The mean dispersal distance of the two-sided power distribution (27) is $b /(1+n)$. Thus, when the dispersal kernels for both sexes have the same shape parameter $n$, we have

$$
\psi=\log _{10}\left(\frac{b_{\mathrm{m}}}{b_{\mathrm{f}}}\right) .
$$

Positive values of $\psi$ indicate male-biased dispersal; negative values indicate female-biased dispersal. 

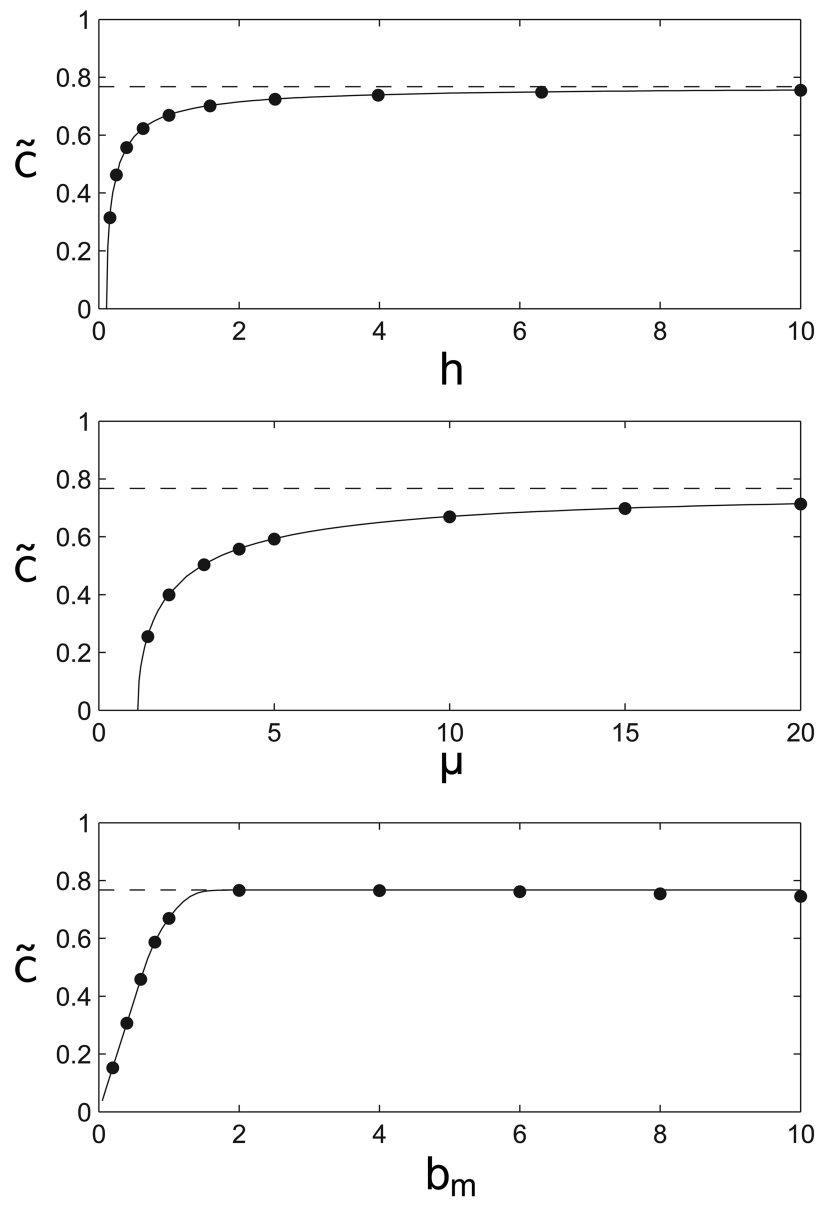

Figure 4: The asymptotic invasion speed, $\tilde{c}$, as calculated via equation (25) (solid curves) and via numerical simulations of model (21) (dots), as a function of the harem size $(h)$, the number of male offspring per mating $(\mu)$, and the maximum male dispersal distance $\left(b_{\mathrm{m}}\right)$. We used the harmonic-mean mating function, the density-dependent survival function (14), and the two-sided power distribution equation (27), with $n=2$ as the dispersal kernel for each sex. The dashed line is the predicted invasion speed of the female-dominant model (12). Except where specified in the graph, we set $a_{\mathrm{m}}=a_{\mathrm{f}}=a=1$, $\mu=10, \phi=10, h=1$, and $b_{\mathrm{m}}=b_{\mathrm{f}}=1$.

In figure 5, we have plotted the invasion speed as a function of dispersal bias $(\psi)$ for the harmonic mean mating function, varying the mating system from monogamous $(h=1)$ to strongly polygynous $(h=100)$. To isolate the effects of bias, we held constant the mean dispersal distance across all individuals,

$$
\delta=\frac{\mu b_{\mathrm{m}}+\phi b_{\mathrm{f}}}{(1+n)(\mu+\phi)},
$$

and kept the sex ratio at birth $1: 1$ (i.e., $\mu=\phi$ ). For comparison, we have also plotted the invasion speed for the standard, female-dominant function.
The results reveal a rich diversity of possible relationships between dispersal bias and invasion speed, depending on the details of local demography. The invasion speed based on the one-sex female-dominant mating function is a monotonically decreasing function of dispersal bias (fig. 5, dashed curve). If males do not matter, then the speed is independent of male dispersal, and the shape of the curve reflects the fact that females move farther, on average, with decreasing values of dispersal bias $\psi$ when the mean dispersal distance $\delta$ is fixed.

When the mating function involves both sexes, however, the effects of dispersal bias on invasion speed can be complex. For the monogamous harmonic mean mating function $(h=1)$, the invasion speed is a symmetric function of dispersal bias, with two peaks at moderate levels of dispersal bias (fig. 5). Beyond these peaks, dispersal bias in either direction reduces the invasion speed of a monogamous invader. The speed decreases asymptotically to 0 as dispersal becomes strongly sex biased and the operational sex ratio at the leading edge of the invasion becomes dominated by one sex. As the mating system becomes increasingly polygynous (greater $h$ ), females dominate local population dynamics and the invasion speed approaches that of the female-dominant mating function, decreasing for positive $\psi$ and increasing for negative $\psi$. Thus, moderate levels of female-biased dispersal can increase the invasion speed of a polygynous invader. Nevertheless, even for large harem sizes, large differences remain between the speeds of the female-dominant and polygynous-harmonic mating functions when dispersal is strongly female biased. These differences occur because a scarcity of males limits population growth at the invasion's leading edge for the two-sex mating function but not the female-dominant function.

The form of the mating function can affect these results. In figure 6 , we use the generalized weighted power mean function (8) and vary the parameter $p$ from the geometric mean $(p \rightarrow 0)$ to the minimum $(p \rightarrow-\infty)$ mating functions. For the monogamous minimum mating function (11), the speed is maximized when dispersal bias is 0 . The opposite is true for the geometric mean mating function, for which (for any harem size) the invasion speed is minimized when dispersal bias is 0 . For intermediate values of $p$, the invasion speed peaks at moderate values of dispersal bias $(\psi)$ and declines to 0 at extreme values, as in figure 5 .

The peaks in invasion speed at nonzero dispersal bias result from two opposing effects of dispersal bias. Consider the right-hand half of figure $6 a{ }^{3}$ When mean dispersal is fixed, larger dispersal bias implies longer male dispersal

\footnotetext{
${ }^{3}$ By considering male fertility, the same reasoning can be used to understand the peak in speed with negative dispersal bias.
} 


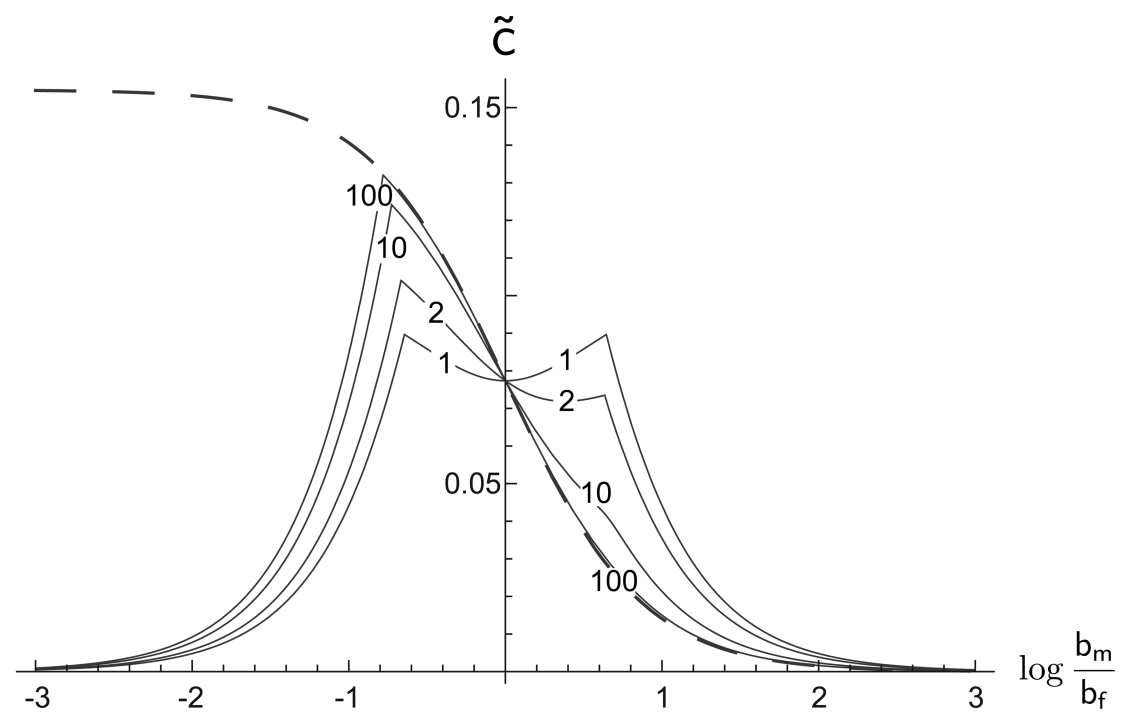

Figure 5: The asymptotic invasion speed, $\tilde{c}$, as calculated via equation (25) as a function of dispersal bias for various harem sizes (curve labels). We used the harmonic mean mating function along with the two-sided power distribution equation (27), with $n=2$ as the dispersal kernel for each sex. We set the mean dispersal distance, $\delta$, to 1/6 (cf. eq. [30]). The dashed curve gives the speed for the female-dominant mating function.

and shorter female dispersal. This increases the operational sex ratio (the ratio $\mathrm{m} / \mathrm{f}$ at mating) at the invasion front. As a result, females become the limiting sex and their dispersal controls the invasion speed. Since female dispersal decreases with increasing bias, this dispersal effect tends to decrease invasion speed. Countering this effect is the fact that under the mating function (8), female fertility - the number of female offspring per female-is a monotonically increasing function of the operational sex ratio for finite $p \leq 0$ (fig. 7). This fertility effect tends to increase the invasion speed.

For small positive dispersal bias, the fertility effect is stronger than the dispersal effect and invasion speed is larger than it would be if both sexes had identical dispersal. For most values of $p$, female fertility has a finite upper bound (fig. 7), and so does the fertility effect on invasion speed. Thus, when dispersal bias is large enough, the dispersal effect is stronger and the invasion speed is smaller than it would be if both sexes had identical dispersal. The locations of the peaks in invasion speed vary with $p$ (fig. 6) because the operational sex ratio at which female fertility reaches its upper bound also varies with $p$ (fig. 7). As $p$ becomes more negative, per capita fertility is essentially maximized at smaller values of the operational sex ratio, and consequently, the invasion speed is maximized at lower absolute values of dispersal bias. For the minimum mating function $(p \rightarrow-\infty)$, the positive effect of sex ratio skew on fertility disappears, and so do the peaks. Under the geometric mean mating function (9), female fertility grows without limit with the operational sex ratio. ${ }^{4}$ In this case, the fertility effect is always stronger than the dispersal effect.

\section{Discussion}

Models of population spatial spread are important tools for understanding and predicting the range dynamics of invasive organisms. Invasions by dioecious species have been analyzed using one-sex or female-dominant models (e.g., Andow et al. 1990, Tinker et al. 2008; Miller and Tenhumberg 2010); such analyses require assumptions about sex-specific dispersal behavior and the importance of both sexes for local population growth. The main contributions of this article, which help to obviate the need for these restrictive assumptions, are threefold.

First, we have developed a spatially explicit sex-structured model for population growth and spread. Our approach is based on integrodifference equations. Other approaches to modeling spatial two-sex population dynamics are possible, including reaction-diffusion equations (Ashih and Wilson 2001) and individual-based models (South and Kenward 2001). We chose to use integrodifference equation models because they have a number of strengths. First, they allow for a wider variety of dispersal distributions than can be accommodated by diffusion-based models

\footnotetext{
${ }^{4}$ This unlimited growth in fertility argues against the use of the geometricmean mating function in invasion models.
} 

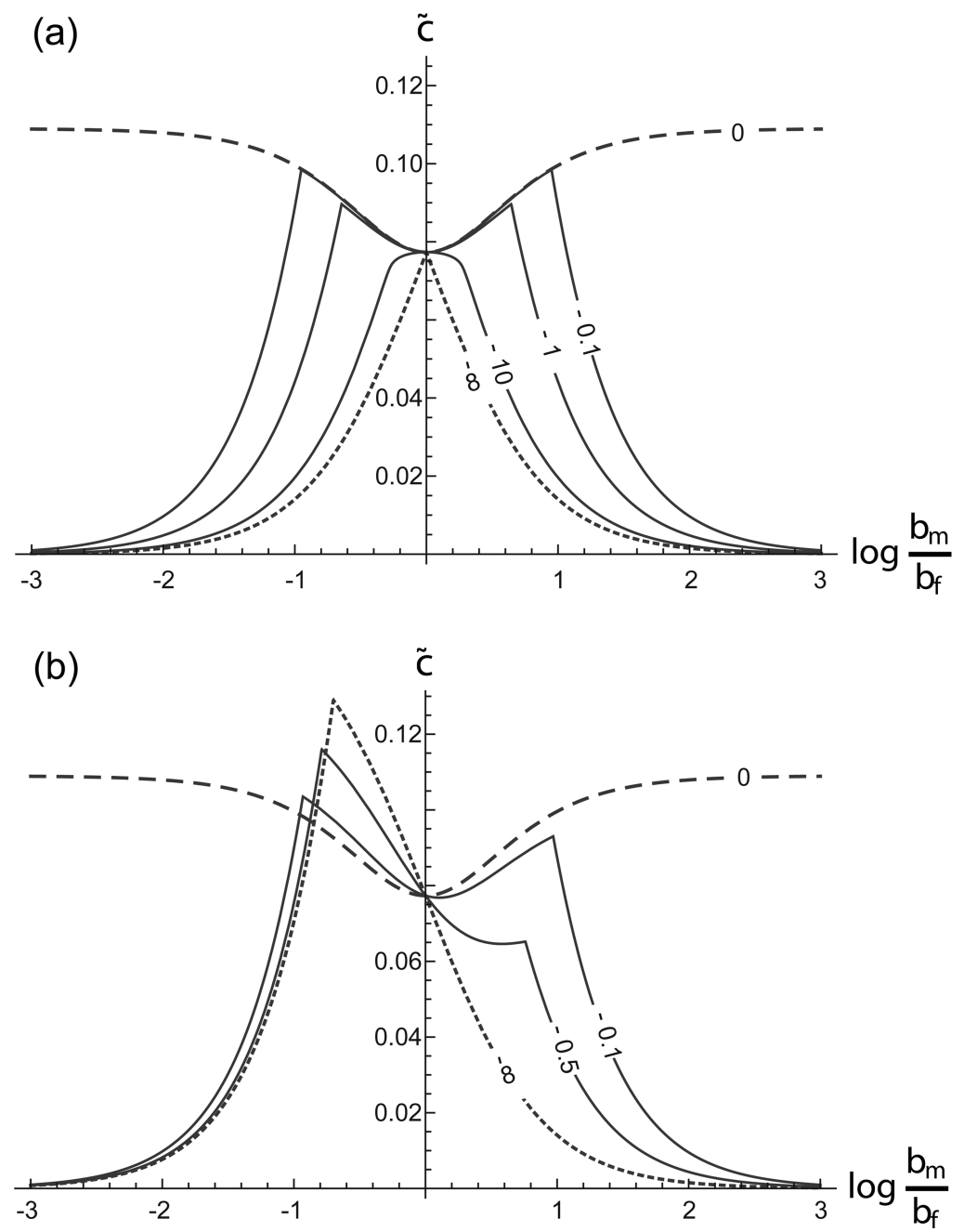

Figure 6: The asymptotic invasion speed, $\tilde{c}$, as calculated via equation (25) as a function of dispersal bias for two mating systems: $(a)$ monogamy $(h=1)$ and $(b)$ polygyny $(h=10)$. We used the two-sided power distribution equation (27) with $n=2$ as the dispersal kernel for each sex and set the mean dispersal distance, $\delta$, to $1 / 6$ (cf. eq. [30]). In each plot, the curves are labeled with the value of $p$ in the weighted power mean mating function (8); we set the weight $w=1 / 2$. The dashed curves $(p=0)$ are the speeds for the geometric mean mating function (9). The dotted curves $(p \rightarrow-\infty)$ are the speeds for the minimum mating function (11). Note that $p=-1$ corresponds to the harmonic mean mating function (fig. 5).

(Neubert et al. 1995). Further, they couple nicely with the matrix population models (Caswell 2001) that are frequently used to describe populations whose vital rates depend on both developmental stage and sex (Pollak 1986). Since measured dispersal distributions also frequently depend on both stage and sex, we hope to incorporate more demographic structure into future models. An approach based on matrix integrodifference equation models (Neubert and Caswell 2000) seems natural.

Second, we derived an explicit formula for the asymptotic velocity of two-sex biological invasions. To our knowledge, this is the first such derivation. We derived this expression by constructing traveling wave solutions to a nonlinear approximation, valid for small population sizes. This contrasts with the derivation of invasion speed in single-sex models, which typically is based on a linear approximation. As a result, the formulas for the singlesex (or female-dominant) speed (4) and the two-sex invasion speed (25) appear different. However, the application of both formulae to empirical systems is straightforward (see Hastings et al. 2005 for a review of applications of one-sex invasion models).

Third, we used the wave speed derivation to evaluate the effects of sex-biased dispersal on the velocity of spatial 


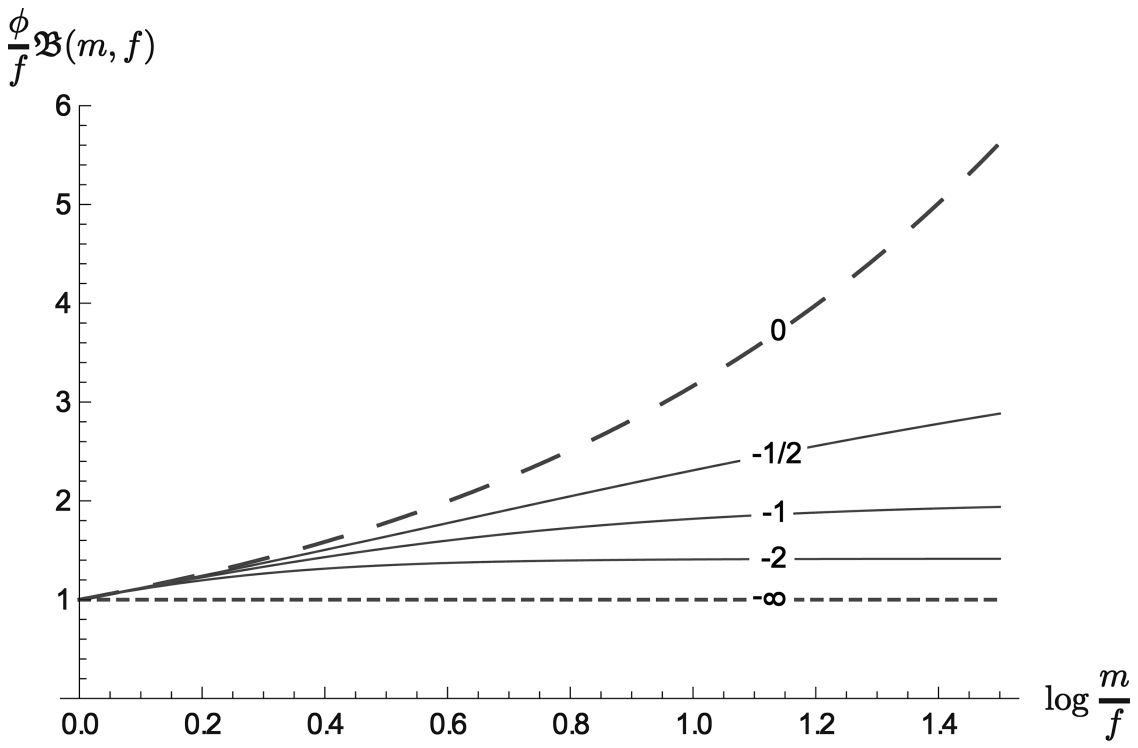

Figure 7: Female fertility $((\phi / f) \mathfrak{B}(m, f))$ as a function of the base 10 logarithm of the operational sex ratio $(m / f)$ for various mating functions of the form of mating function (8). The curves are labeled with the value of $p$; we set the weight $w=1 / 2$. The dashed curve $(p=0)$ is the female fertility for the geometric mean mating function $(9)$. The dotted curve $(p \rightarrow-\infty)$ is the female fertility for the minimum mating function (11). Note that $p=-1$ corresponds to the harmonic mean mating function (10).

spread. While sex-biased dispersal is common in nature, this is to our knowledge the first analysis of its dynamic consequences in the context of biological invasions. Our results confirm that when dispersal distances are equal between the sexes, one-sex theory yields accurate predictions for the dynamics of two-sex invasions. However, any difference in dispersal between the sexes causes incipient populations near the invasion front to deviate from the birth sex ratio. As in one-sex invasions, the velocity of two-sex invasions is strongly influenced by the rate of increase at the low-density leading edge. Thus, perturbations to the operational sex ratio caused by dispersal bias can yield a diverse set of possible outcomes not predicted by classic invasion theory. We find that sex bias in dispersal can produce invasion speeds that are either higher or lower than those predicted by one-sex models. Further, sexbiased dispersal can lead to invasion speeds that asymptotically approach 0 when one-sex models (with all else equal) would predict a positive invasion speed. We also found that for two-sex models, the initial distribution of males and females may determine whether an invasion will materialize at all.

Whether sex-biased dispersal accelerates, decelerates, or virtually halts an invasion depends on complex interactions among the direction of bias, the magnitude of bias, the social mating system, and the form of the mating function. Numerous mating functions have been proposed in the demography literature (Caswell 2001). The har- monic mean mating function is generally regarded as the most realistic (Caswell and Weeks 1986; Lindstrom and Kokko 1998; Ranta et al. 1999). However, we are aware of no studies that have compared the fits of alternative mating functions to empirical data. Given the importance of the mating function for spread dynamics, there is clearly a need for studies that confront candidate models of twosex demography with data. Experimental perturbations to the operational sex ratio are a potentially valuable approach for elucidating population growth dynamics at the leading edge of a two-sex invasion.

In conclusion, our results indicate that sex differences in dispersal should be assessed before one-sex models can be applied to dioecious invaders. Where sex differences occur, our results further suggest that parameters governing local, two-sex demography should be estimated in order to generate accurate predictions for rates of spatial spread. We are optimistic that integration of the two-sex theoretical framework developed here with empirical data will advance understanding and management of invasions by dioecious organisms.

\section{History}

The two-sex models constructed in this manuscript were first analyzed by A. K. Shaw and M. G. Neubert and presented at the Ecological Society of America annual meeting in 2004, where A. K. Shaw won the Lotka-Volterra Prize 
for best student poster from the Theoretical Ecology Section. The models were subsequently independently derived by T. E. X. Miller and B. D. Inouye. When we each became aware of each other's results, we agreed to combine our previously and separately prepared papers into this manuscript.
Bishop. 2005. When can herbivores slow or reverse the spread of an invading plant? a test case from Mount St. Helens. American Naturalist 166:669-685.

Fisher, R. 1930. The genetical theory of natural selection. Clarendon, Oxford.

Franklin, A. B., D. R. Anderson, E. D. Forsman, K. P. Burnham, and F. W. Wagner. 1996. Methods for collecting and analyzing demographic data on the northern spotted owl. Avian Biology 17: $12-20$.

$\rightarrow$ Greenwood, P. 1980. Mating systems, philopatry and dispersal in birds and mammals. Animal Behaviour 28:1140-1162.

\section{Acknowledgments}

We thank K. Anderson, H. Caswell, C. Lee, and M. Simon for helpful discussions and suggestions. This work was supported in part by a Woods Hole Oceanographic Institution Summer Student Fellowship and a National Sci $\rightarrow$ ence Foundation (NSF) Graduate Research Fellowship to A.K.S., USDA grants to T.E.X.M. (CSREES-2007-02270), and NSF grants to M.G.N. (DEB-0235692) and to B.D.I. (DEB-0816838). T.E.X.M. was also supported by the Rice University Huxley Fellowship; M.G.N. also acknowledges support from the Andrew W. Mellon Foundation Endowec' Fund for Innovative Research.

Hadeler, K. 1989. Pair formation in age-structured populations. Acta Applicandae Mathematicae 14:91-102.

Hardy, G., J. E. Littlewood, and G. Polya. 1952. Inequalities. 2nd ed. Cambridge University Press, Cambridge.

Hastings, A., K. Cuddington, K. Davies, C. Dugaw, S. Elmendorf, A. Freestone, S. Harrison, et al. 2005. The spatial spread of invasions: new developments in theory and evidence. Ecology Letters 8:91101.

Iannelli, M., M. Martcheva, and F. A. Milner. 2005. Gender-structured population modeling: mathematical methods, numerics, and simulations. SIAM, Philadelphia.

Keitt, T., M. Lewis, and R. Holt. 2001. Allee effects, invasion pinning, and species' borders. American Naturalist 157:203-216. $\rightarrow$ Kot, M. 1992. Discrete-time travelling waves: ecological examples. Journal of Mathematical Biology 30:413-436.

\section{Literature Cited}

Abramowitz, M., and I. A. Stegun, eds. 1972. Handbook of math $\rightarrow$ ematical functions with formulas, graphs, and mathematical tables. Dover, New York.

$\rightarrow$ Andow, D., P. Kareiva, S. Levin, and A. Okubo. 1990. Spread of invading organisms. Landscape Ecology 4:177-188.

$\rightarrow$ Ashih, A. C., and W. G. Wilson. 2001. Two-sex population dynamics in space: effects of gestation time on persistence. Theoretical Population Biology 60:93-106.

$\rightarrow$ Buckley, Y., E. Brockerhoff, L. Langer, N. Ledgard, H. North, and M. Rees. 2005. Slowing down a pine invasion despite uncertainty in demography and dispersal. Journal of Applied Ecology 42:10201030.

Bullen, P. S. 2003. Handbook of means and their inequalities. Kluwer Academic, Dordrecht.

Caswell, H. 2001. Matrix population models: construction, analysis, and interpretation. 2nd ed. Sinauer, Sunderland, MA.

$\rightarrow$ Caswell, H., and D. Weeks. 1986. Two-sex models: chaos, extinction, and other dynamic consequences of sex. American Naturalist 128 . 707-735.

$\rightarrow$ Clarke, A., B. E. Sæther, and E. Roskaft. 1997. Sex biases in avian dispersal: a reappraisal. Oikos 79:429-438.

$\rightarrow$ Crozier, L., and G. Dwyer. 2006. Combining population-dynamic and ecophysiological models to predict climate-induced range shifts. American Naturalist 167:853-866.

$\rightarrow$ Dale, S. 2001. Female-biased dispersal, low female recruitment, unpaired males, and the extinction of small and isolated bird populations. Oikos 92:344-356.

$\rightarrow$ Dobson, F. 1982. Competition for mates and predominant juvenil $\rightarrow$ dispersal in mammals. Animal Behavior 30:1183-1192.

$\rightarrow$ Fagan, W., M. Lewis, M. Neubert, and P. van den Driessche. 2002. Invasion theory and biological control. Ecology Letters 5:148-157.

$\rightarrow$ Fagan, W., M. Lewis, M. Neubert, C. Aumann, J. Apple, and J. G $\rightarrow$ Olsson, M., and R. Shine. 2003. Female-biased natal and breeding plied Mathematics Quarterly 10:139-170.

Kot, M., and W. M. Schaffer. 1986. Discrete-time growth-dispersal models. Mathematical Biosciences 80:109-136.

$\rightarrow$ Kot, M., M. Lewis, and P. van den Driessche. 1996. Dispersal data and the spread of invading organisms. Ecology 77:2027-2042.

Kotz, S., and J. Van Dorp. 2004. Beyond beta: other continuous families of distributions with bounded support and applications. World Scientific, Singapore.

$\rightarrow$ Legendre, S., J. Clobert, A. Møller, and G. Sorci. 1999. Demographic stochasticity and social mating system in the process of extinction of small populations: the case of passerines introduced to New Zealand. American Naturalist 153:449-463.

$\rightarrow$ Lindstrom, J., H. Kokko. 1998. Sexual reproduction and population dynamics: the role of polygyny and demographic sex differences. Proceedings of the Royal Society B: Biological Sciences 265:483488.

$\rightarrow$ Maron, J., and S. Harrison. 1997. Spatial pattern formation in an insect host-parasitoid system. Science 278:1619-1621.

$\rightarrow$ Miller, T., and B. Tenhumberg. 2010. Contributions of demography and dispersal parameters to the spread of a stage-structured insect invasion. Ecological Applications 20:620-633.

$\rightarrow$ Neubert, M., and H. Caswell. 2000. Demography and dispersal: calculation and sensitivity analysis of invasion speed for structured populations. Ecology 81:1613-1628.

$\rightarrow$ Neubert, M., M. Kot, and M. Lewis. 1995. Dispersal and pattern formation in a discrete-time predator-prey model. Theoretical Population Biology 48:7-43.

$\rightarrow$ Neubert, M., and I. Parker. 2004. Projecting rates of spread for invasive species. Risk Analysis 24:817-831.

Okubo, A. 1980. Diffusion and ecological problems: mathematical models. Biomathematics 10. Springer, Berlin. 
dispersal in an alpine lizard, Niveoscincus microlepidotus. Biologica Journal of the Linnean Society 79:277-283.

$\rightarrow$ Pollak, R. A. 1986. A reformulation of the two-sex problem. Demography 23:247-259.

$\rightarrow$ Potapov, A. B., and M. Lewis. 2004. Climate and competition: the effect of moving range boundaries on habitat invasibility. Bulletin of Mathematical Biology 66:975-1008.

$\rightarrow$ Rankin, D., and H. Kokko. 2007. Do males matter? the role of males in population dynamics. Oikos 116:335-348.

$\rightarrow$ Ranta, E., V. Kaitala, and J. Lindstrom. 1999. Sex in space: population dynamic consequences. Proceedings of the Royal Society B: Biological Sciences 266:1155-1160.

$\rightarrow$ Rosen, K. 1983. Mathematical models for polygynous mating systems Mathematical Modelling 4:27-39.

$\rightarrow$ Schoen, R. 1983. Measuring the tightness of a marriage squeeze Demography 20:61-78.

$\rightarrow$ Sharov, A. A., A. M. Liebhold. 1998. Bioeconomics of managing the spread of exotic pest species with barrier zones. Ecological Ap plications 8:833-845.

$\rightarrow$ Skellam, J. 1951. Random dispersal in theoretical populations. Biometrika 38:196-218.

Smith, M. H. 1968. Dispersal of the old-field mouse, Peromyscus polionotus. Bulletin of the Georgia Academy of Science 26:45-51.
South, A., and R. Kenward. 2001. Mate finding, dispersal distances, and population growth in invading species: a spatially explicit model. Oikos 95:53-58.

$\rightarrow$ Tinker, M., D. Doak, and J. Estes. 2008. Using demography and movement behavior to predict range expansion of the southern sea otter. Ecological Applications 18:1781-1794.

Van Dorp, J., and S. Kotz. 2002. The standard two-sided power distribution and its properties: with applications in financial engineering. American Statistician 56:90-99.

Weinberger, H. 1978. Asymptotic behavior of a model of population genetics. Pages 47-98 in J. Chadam, ed. Nonlinear partial differential equations and applications. Vol. 648. Lecture Notes in Mathematics. Springer, New York.

- 1982. Long-time behavior of a class of biological models. SIAM Journal on Mathematical Analysis 13:353.

Wilson, W., S. Harrison, A. Hastings, and K. McCann. 2002. Exploring stable pattern formation in models of tussock moth populations. Journal of Animal Ecology 68:94-107.

Zhou, Y. and Kot, M. 2010. Discrete-time growth-dispersal models with shifting species ranges. Theoretical Ecology 4:13-25.

Associate Editor: Benjamin M. Bolker Editor: Mark A. McPeek

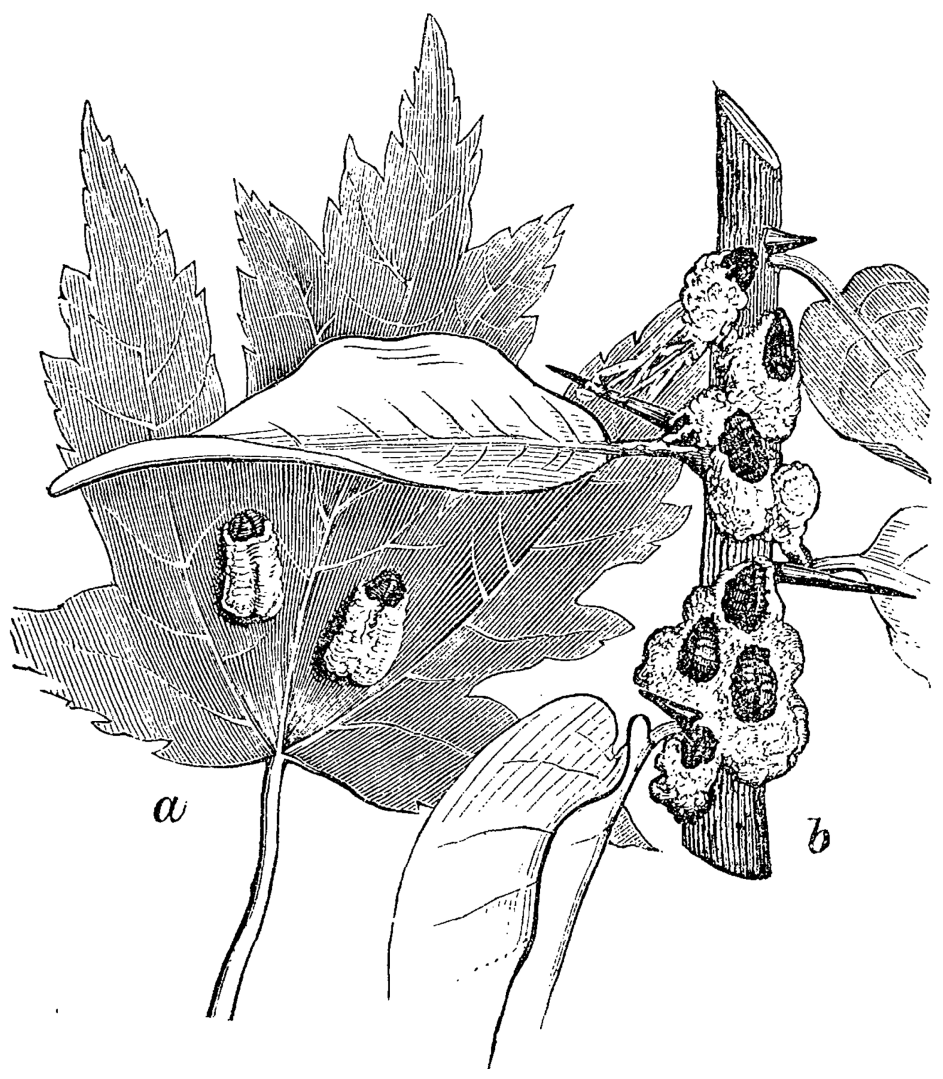

a, Lecanium on maple; b, Lecanium on osage orange. From "The Maple-Tree Bark-Louse" by Emily A. Smith (American Naturalist, 1878, 12:655-661). 